\title{
Investigation of Electrical Properties \& Mechanical Quality Factor of Piezoelectric Material (PZT-4A)
}

\author{
Zubair Butt ${ }^{\dagger}$, Zeeshan Anjum*, Amir Sultan**, Faisal Qayyum*, \\ Hafiz Muhammad Khurram Ali** and Shahid Mehmood **
}

\begin{abstract}
Piezoelectricity is the capability of a piezoelectric material to change mechanical energy into electrical energy. The determination of electrical and mechanical properties plays a significant role in characterizing the piezoelectric material. The energy losses characteristics of piezoelectric material can be described by mechanical quality factor. In this paper, the output voltage and mechanical quality factor of Lead Zirconate Titanate (PZT-4A) piezoelectric material is determined under various resistance and loading conditions by using the test setup. The commercial FEM software ABAQUS is used to analyze the performance of piezoelectric material under static loading conditions. It is observed that these properties affect the performance of a material particularly in the designing of smart structures. The experimental results are partially compared to the simulation values.
\end{abstract}

Keywords: Piezoelectricity, PZT-4A, Mechanical quality factor, Finite element method

\section{Introduction}

Energy harvesting is the process in which energy is acquired from the external sources and transformed into usable electrical energy or energy is captured or stored for future use. Among the three general mechanisms of energy harvesting through vibrations (electrostatics, electromagnetic and piezoelectric) piezoelectric materials have a superior performance and can be fabricated into different scales: macro, micro and nano [1]. Direct piezoelectricity (the generation of electrical voltage by applying mechanical stress) and inverse piezoelectricity are the two types of piezoelectric effects. Jacques Curie and Pierre Curie discovered piezoelectricity in 1880 [2]. The energy harvesting through piezoelectricity uses direct piezoelectric effect. Piezoelectric materials can be utilized to obtain voltages of very small value and hence can drive low voltage devices. The finite element analysis (FEA) is an efficient method to simulate and analyse the structural and electrical characteristic of piezoelectric material [3]. In the previous decades, a lot of research has been done in the field of energy harvesting. The enhancement in this research has revolutionized in wireless technology and electronic devices like Micro-Electro-Mechanical Systems (MEMS) [4].

The aim of this research work is to investigate the effect of varying resistance on the output voltage of Lead Zirconate Titanate (PZT-4A) at different frequencies using

$\dagger$ Corresponding Author: Dept. of Mechatronics Engineering, UET Taxila, Chakwal Campus, Pakistan. (zubairbutt64@yahoo.com).

* Dept. of Mechanical Engineering, UET Taxila, Pakistan.

(zeeshan-89@hotmail.com, faisal.qayyum@uettaxila.edu.pk)

** Dept. of Mechatronics Engineering, UET Taxila, Chakwal Campus,

Pakistan. (\{amir.sultan, khurram.ali, shahid.mehmood\}@uettaxila.edu.pk)

Received: April 19, 2016; Accepted: October 31, 2016 the proposed experimental setup. Effect of loads on the mechanical quality factor is also analyzed. Furthermore the experimental results are compared with numerical simulation using standard FEA software; ABAQUS.

\section{Historical Background}

Piezoelectric effect based energy harvesting devices that transforms mechanical energy into electrical energy are a very prestigious energy source for remote sensing and embedded devices. Piezoelectric materials are the fundamental elements in electro-mechanical equipment and play a significant role in smart structure [5]. In 1947 a process was developed in which mechanical energy is converted into electrical energy with the help of piezoelectric vibrator [6]. First experimental study to harvest energy by using piezoelectric material was carried out in 1984 [7]. In 1992 Schmidt also conducted an experimental investigation to study the behavior of polyvinylidene difluoride (PVDF) specimen at various high and low frequency amplitude [8]. The first work to harvest energy from the human body by using piezoelectric material was published in 1995 . The aim was to derive the low power electronics devices by using piezoelectric energy harvesting system [9]. An experimental and mathematical frequency domain analysis for two degree of freedom wing-based piezoelectric energy harvester was conducted to study the effect of electrical load resistance at various frequencies and amplitudes [10]. In 2014 an experimental investigation was carried out to harvest energy by using piezoelectric generator over wide range of frequency and optimal load resistance [11]. Mechanical quality factor helps in designing of a sensor 
and actuator and it is one of the most important parameter to determine the energy loss characteristic of piezoelectric material $[12,13]$.The range of the mechanical quality factor $\left(\mathrm{Q}_{m}\right)$ varies from 3700 at $158 \mathrm{kHz}$ frequency to 930 at $1.18 \mathrm{MHz}$ frequency [14]. A study reported a new class of piezoelectric materials having high mechanical quality factor which vibrate at $1.7 \mathrm{MHz}$ and have a lot of applications as sensor and actuator [15]. Up till now the development of piezoelectric transducers was mainly based on trial and error which is not only time consuming as well as much expensive. This kind of development is not aligned with modern industrial engineering practices, which is to assist development by computer simulation software for the theoretical prediction of properties expected to results from a given transducer design. Recently finite element analysis (FEA) has become vital tool for researchers for the analysis of mechanical components as well as piezoelectric sensors and actuators [16-18]. As we know, the properties of piezoelectric materials are very important to the design of MEMS sensors and actuators. The FEA software is used to simulate the structure of piezoelectric devices successfully and accurately [19].

The prediction of electrical and mechanical properties plays a vital role in characterising the piezoelectric material and they are influenced by operating and testing conditions [20]. In this study, our aim is to investigate the mechanical characteristic and electrical response of a rectangular shaped Lead Zirconate Titanate (PZT-4A) piezoelectric material simultaneously. The work has been divided in four major phases. Phase-1 determines the output voltage at various resistance and frequency conditions. Phase-2 determines the output voltage of a piezoelectric material at various resistance and loading conditions. The energy loss characteristic and the effect of loading on the mechanical quality factor are analysed in phase-3. In phase-4 finite element simulation was carried out using numerical package ABAQUS ${ }^{\mathrm{TM}} /$ Standard to determine the effect of loading on the output voltage of Lead Zirconate Titanate (PZT-4A) piezoelectric material.

\section{Experimental Setup}

The experimentation was performed to determine the effect of various parameters on peak DC voltage and mechanical quality factor of a piezoelectric material. A rectangular shaped Lead Zirconate Titanate (PZT-4A) piezoelectric material was used and the parent properties of the specimen as provided by the supplier are given in Table 1. The size of specimen was $10 \times 20 \times 5 \mathrm{~mm}$. The experimental setup consisted of a load cell (model HYTEK) placed on mild-steel iron sheet and a screw mechanism connected with $12 \mathrm{~V}$ DC motor for regulating the load. A DC motor can apply a maximum of $600 \mathrm{kN} / \mathrm{m}^{2}$ load under wide frequency range of $1-10 \mathrm{kHz}$. The copper
Table 1. Properties of specimen provided by the supplier

\begin{tabular}{c|c}
\hline Description & Value \\
\hline Density $(\rho)$ & $7800 \mathrm{~kg} / \mathrm{m}^{3}$ \\
\hline Young modulus $(\mathrm{E})$ & $5.5 \times 10^{10} \mathrm{~N} / \mathrm{m}^{2}$ \\
\hline Mechanical Quality factor $\left(\mathrm{Q}_{m}\right)$ & 75 \\
\hline Piezoelectric voltage coefficient $\left(\mathrm{g}_{33}\right)$ & $24 \times 10^{-3}$ Volt metres/Newton \\
\hline Piezoelectric strain coefficient $\left(\mathrm{d}_{33}, \mathrm{~d}_{31}\right)$ & $374 \times 10^{-12},-171 \times 10^{-12} \mathrm{~m} / \mathrm{volt}$ \\
\hline Dielectric constant $\left(\varepsilon_{33}{ }^{\mathrm{T}}\right)$ & $1.504 \times 10^{-8} \mathrm{~F} / \mathrm{m}$ \\
\hline Elastic compliance constant $\left(\mathrm{S}_{11}{ }^{\mathrm{E}}\right)$ & $16.4 \times 10^{-12} \mathrm{~m}^{2} / \mathrm{N}$ \\
\hline Initial depolarization field $\left(\mathrm{E}_{\mathrm{c}}\right)$ & $5 \times 10^{5} \mathrm{~V} / \mathrm{m}$ \\
\hline Polarization field $\left(\mathrm{E}_{\mathrm{p}}\right)$ & $2 \times 10^{6} \mathrm{~V} / \mathrm{m}$ \\
\hline Curie Temperature & $350{ }^{\circ} \mathrm{C}$ \\
\hline
\end{tabular}

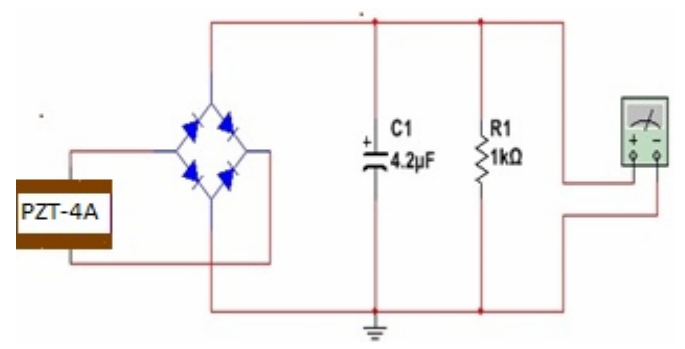

Fig. 1. Energy harvesting circuit

electrodes which acted as anode and cathode were located at upper and lower end of a specimen. Piezoelectric material normally gives peak to peak $\mathrm{AC}$ voltage under vibration. For practical applications DC voltage is needed to drive any system. So in our case, an energy harvesting circuit consisted of bridge diodes (D5SBA) and a resistor connected with copper electrodes as shown in Fig. 1 was included in test setup to measure the peak DC voltage across a $4.2 \mu \mathrm{F}$ capacitor. The capacitance was noted via capacitance meter (DM-6013L). Specific resistance required according to the condition of circuit was provided by the decade resistor box (RM6-10073) having two crocodile probes connected in series with a test specimen. An impedance analyzer (E4990A) having frequency range of $12 \mathrm{~Hz}$ to $120 \mathrm{MHz}$ was used for measuring impedance. To electrically shock the specimen with sinusoidal waves of different frequencies, the function generator (HM-8150) was used. The test setup was placed on an isolated bench to avoid any intervention of noise from surrounding environment. The output response was investigated on digital oscilloscope (GPS-1072B) under varying resistance at multiple frequencies and loading conditions. The measurement locations are shown in Fig. 2

Resonating frequency $\left(f_{a}\right)$ and anti-resonating frequency $\left(f_{b}\right)$ was determined by a circuit method as shown in Fig. 3 . The capacitance (C) was determined via capacitance meter and the mechanical quality factor $\left(\mathrm{Q}_{m}\right)$ was calculated by using Eqs. (1) and (3)

$$
\mathrm{Q}_{m}=\frac{\mathrm{Xc}}{\mathrm{R}}
$$

As impedance $X_{c}$ is given by 


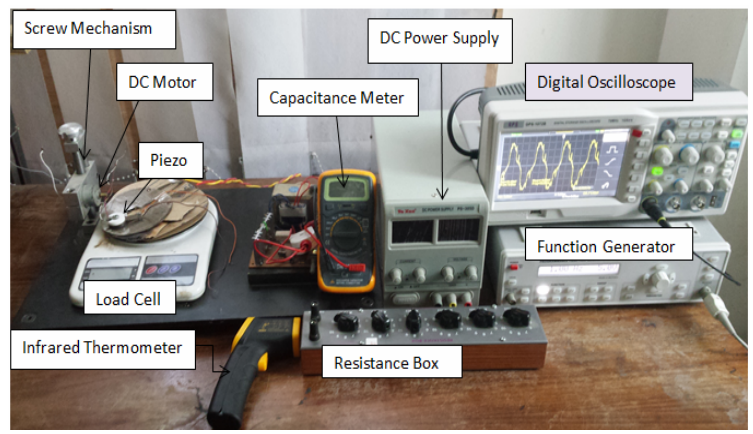

Fig. 2. Experimental setup of the proposed system

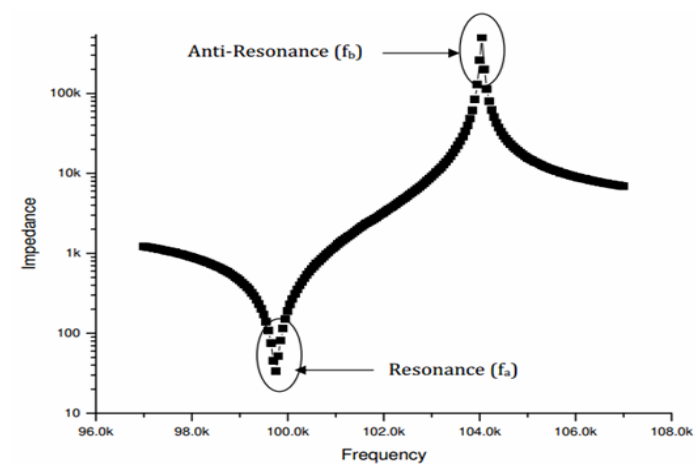

Fig. 3. Resonance and anti-resonance frequency in the impedance curve of piezoelectric material [21].

$$
X_{c}=\frac{1}{2 \pi \mathrm{fC}}
$$

So (1) becomes

$$
\mathrm{Q}_{m}=\frac{1}{2 \pi \Delta \mathrm{fRC}}
$$

where $\Delta f=f_{b}-f_{a}$

A recently proposed electrical power method for calculating mechanical quality factor showed that the maximum mechanical quality factor occurs between resonance and anti-resonance frequencies while the previous methods (impedance and burst) were limited to provide the mechanical quality factor only at resonance and anti-resonance frequencies [22-24].

\section{Results and Discussion}

In this section simulation and experimental observations, results obtained are discussed and interpreted.

\subsection{Phase-1: Effect of Resistance on Peak DC voltage at various frequency conditions}

In this phase the effect of resistance on peak DC voltage was determined at various frequency conditions. The function generator connected with decade resistor box

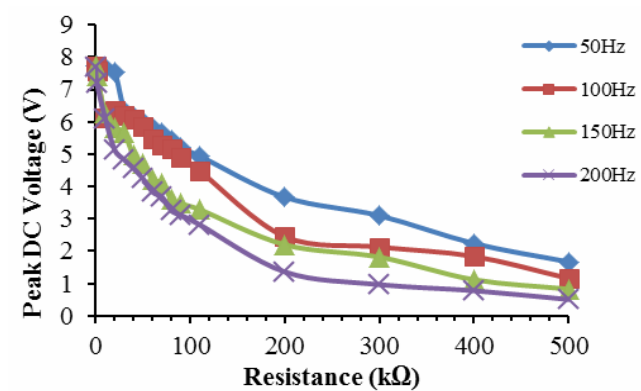

Fig. 4. Decrease in peak DC voltage with increase in resistance and frequency

has been used for providing a series of sinusoidal signals of different frequencies under variable resistor loading conditions. With respect to resistance change, the linear behavior of specimen was revealed as shown in Fig. 4. It was observed that the output voltage decays with the increase in values of resistance. With the variation of frequency, the resistance changes and so the voltage decreases when the frequency increases. It is determined from results that with the change in frequency and resistance, the output voltage changes greatly. When the frequency is maximum voltage drop is also maximum.

The maximum drop in voltage was observed at $500 \mathrm{k} \Omega$ resistances across a $200 \mathrm{~Hz}$ frequency. It was observed that output voltage of the harvester increases with the increase in impedance of the resistor while resonance frequency decreases. The resonance frequency of the harvester used was $180 \mathrm{~Hz}$ which is also equal to its natural frequency. So when the harvester vibrated near its resonance frequency, its output voltage increased otherwise decreased with the increase in load resistance. But as the frequency of the harvester exceeded the resonance frequency, the output voltage dropped due to depolarization effect.

The analysis of results obtained from the experiment describe that the current results are somewhat better than previous published data by R. A Pasha et al. [21].

\subsection{Phase-2: Effect of Resistance on Peak DC voltage at various loading conditions}

Fig. 5 shows the relationship of peak DC voltage and resistance at various loading conditions. The experiment was performed at $20,40,60,80$ and $100 \mathrm{kN} / \mathrm{m}^{2}$ under variable resistance ranging from $1 \mathrm{k} \Omega$ to $15 \mathrm{k} \Omega$ across a $5 \mathrm{~Hz}$ frequency. It was observed that on increasing resistance and loading, the peak DC voltage increases. It should be noted that dynamic series of applied load is directly responsible for output voltage. The output voltage increases with the increase of external resistance attached across the harvester. The increase in the resistance is also increase in the power extracted from the harvester because power is a square function of voltage. The total power consumed by the harvester is inversely proportional to the value of external resistor. Since the effect of voltage on 


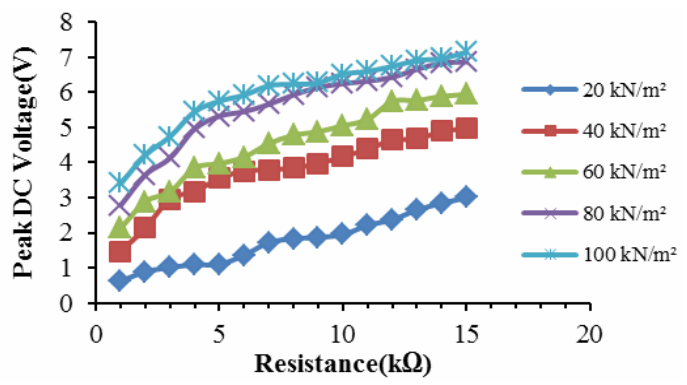

Fig. 5. Increase in peak DC voltage with the increase in resistance and load at $5 \mathrm{~Hz}$ frequency

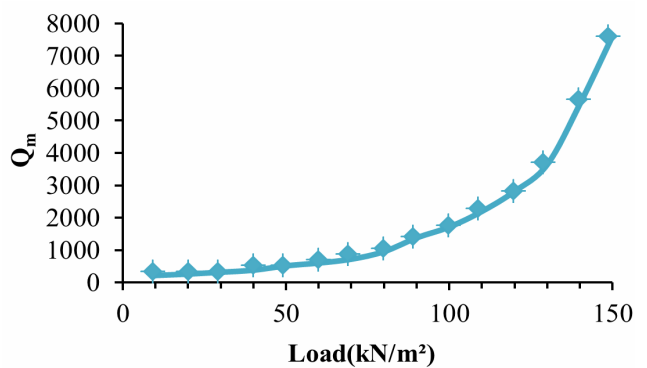

Fig. 6. Increase in mechanical quality factor with the increase in load

power is greater than effect of external resistance, so the net effect on increasing the value of external resistance on extracted voltage or power is positive and the output voltage increases. The same kind of behavior was observed during vibration energy harvesting with a clamped piezoelectric circular diaphragm [5]

\subsection{Phase-3: Prediction of Mechanical Quality Factor $\left(Q_{m}\right)$ under variable Loading conditions}

In this phase experimentation was performed to find out the mechanical quality factor $\left(\mathrm{Q}_{m}\right)$ of a specimen at a constant resistance of $10 \mathrm{k} \Omega$ under variable loading conditions ranging from $10 \mathrm{kN} / \mathrm{m}^{2}$ to $150 \mathrm{kN} / \mathrm{m}^{2}$. Increase in mechanical quality factor has been observed with the increase in load at constant resistance as shown in Fig. 6. With the increase in applied load, the material vibration increases and domain element becomes polarized. In that case the magnitude of the electromechanical resonant strain has been increased. It means that by increasing the load, energy losses decrease (i.e. efficiency increases) and mechanical quality factor increases. The power handling capacity of the harvester is also limited at times with high value of mechanical quality factor by dynamic tensile or compressive strength of $600 \mathrm{kPa}$ which reduces the efficiency due to depolarization and mechanical losses of the harvester.

\subsection{Phase-4: Piezoelectric Static Analysis}

A 3-D solid finite element model was developed in ABAQUS software to accurately predict the static response
Table 2. Properties of PZT-4A [19]

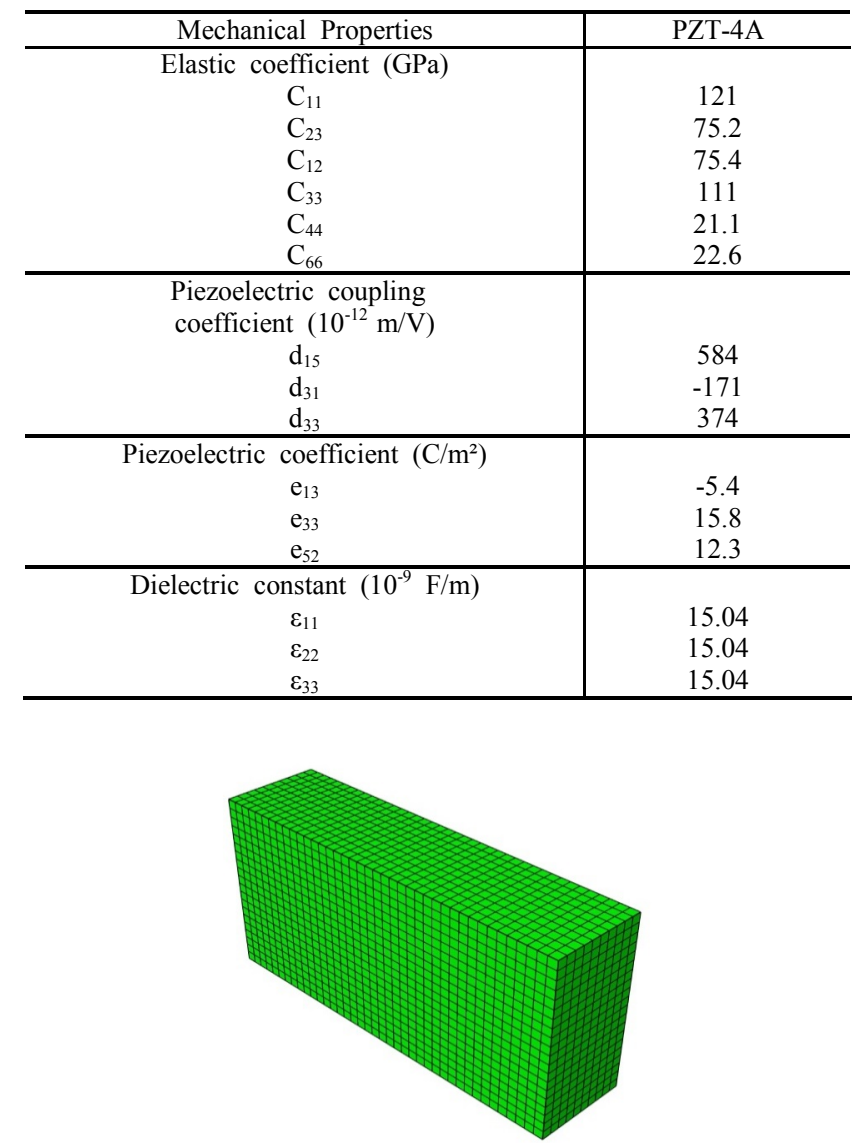

Fig.7. PZT-4A meshed model

of a material subjected to a constant load. A rectangular shaped hexahedral coupled field element C3D8E having dimensions of $10 \times 20 \times 5 \mathrm{~mm}$ was used to simulate the PZT$4 \mathrm{~A}$. The anisotropic material properties used in simulation of PZT-4A are given in Table 2. Meshed model consisted of 78000 hexahedral element and 8874 nodes as shown in Fig. 7. ABAQUS uses Newton's integration scheme for designing the model geometry of the material. A constant load was applied on the top surface of the material along their polarization directions. The design models of piezoelectric materials simulate free expansion of the actuators with fixed boundary condition at one end to avoid the rigid body motion. For mechanical boundary conditions all surface are free and for electrical boundary conditions bottom surface of the material was grounded and zero charge/symmetry condition was considered on the top surface. An initial understanding of performance characteristic of PZT-4A was established through experimentation.

Fig. 8 indicates the output voltage of PZT-4A with the experimented and simulated results. It is examined that the output voltage increases when loading increases. The experimental values are in good agreement with the numerical simulation results with less than 5\% error. Fig. 9 indicates the FEM model of voltage change transmission under constant loading condition. 


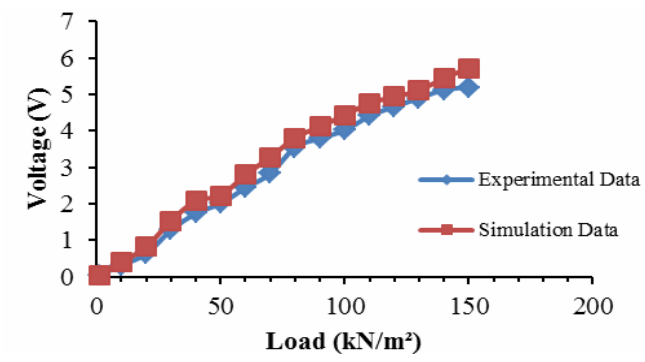

Fig. 8. Comparison of experimental and numerical simulation results under variable loading conditions

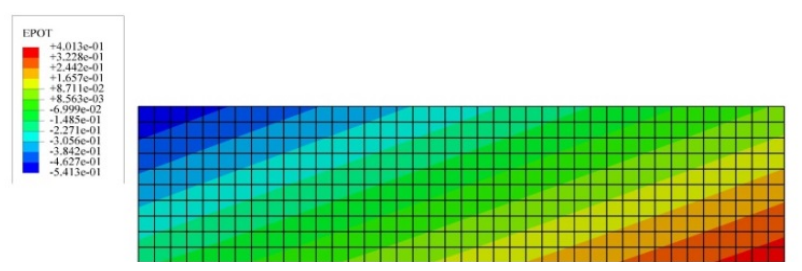

Fig. 9. ABAQUS predicted electrical potential variation in PZT-4A specimen under constant loading

\section{Conclusions}

On the basis of the results obtained from the current research work it was found that

1. With the increase in resistance from $1 \mathrm{k} \Omega$ to $500 \mathrm{k} \Omega$ the output voltage of Lead Zirconate Titanate (PZT-4A) piezoelectric material decreases at different frequency conditions. The maximum voltage drop was observed at $500 \mathrm{k} \Omega$ resistance across a $200 \mathrm{~Hz}$ frequency.

2. The negative linear relationship was observed between resistance and peak DC voltage at different frequency conditions.

3. The output voltage increases with the increase in resistance and load which shows that forceful range of applied load is directly responsible for output voltage.

4. The mechanical quality factor increase when loading increases which shows that efficiency of a piezoelectric material increases.

5. It is observed that piezoelectric material performs well when loading increases. The results from the simulation indicate that FEM model is correct and good for future studies under various loading conditions.

\section{Acknowledgements}

The authors acknowledge the support by the Mechanical Engineering Department of the University of Engineering and Technology Taxila, Pakistan during this research work.

\section{References}

[1] K. Cook-Chennault, N. Thambi and A. Sastry,
Powering MEMS portable devices - a review of non-regenerative and regenerative power supply systems with special emphasis on piezoelectric energy harvesting systems. Smart Materials and Structures, 2008. 17(4): p. 043001.

[2] A. Safari and E.K. Akdogan, Piezoelectric and acoustic materials for transducer applications. Springer Science \& Business Media, 2008.

[3] J. Latalski, Modelling of macro fiber composite piezoelectric active elements in ABAQUS system. Eksploatacja i Niezawodnosc-Maintenance and Reliability, 2011 (4): p. 72-78.

[4] N. Paliwal, N. Mukhija and D. Bhatia, Design and optimization of high quality factor MEMS piezoelectric resonator with pseudo electrodes.in Reliability, Infocom Technologies and Optimization (ICRITO) (Trends and Future Directions), 2015 4th International Conference on. 2015. IEEE.

[5] X. Chen, T. Yang. W. Wang and X. Yao, Vibration energy harvesting with a clamped piezoelectric circular diaphragm. Ceramics International, 2012. 38: p. S271-S274.

[6] W. L. Bond, and W. P. Mason, Piezoelectric vibrator. 1947, Google Patents.

[7] E. Häsler, L. Stein, and G. Harbauer, Implantable physiological power supply with PVDF film. Ferroelectrics, 1984. 60(1): p. 277-282.

[8] V. H. Schmidt, Piezoelectric energy conversion in windmills. in Ultrasonics Symposium, 1992. Proceedings., IEEE 1992. 1992. IEEE.

[9] J. F. Antaki, G. E. Bertocci, E. C. Green, A. Nadeem, T. Rintoul, R. L. Kormos and B. P. Griffith, A gaitpowered autologous battery charging system for artificial organs. ASAIO journal, 1995. 41(3): p. M588-M595.

[10] A. Erturk, WGR Vieira, C. De Marqui Jr. and DJ Inman, On the energy harvesting potential of piezoaeroelastic systems. Applied Physics Letters, 2010. 96(18): p. 184103.

[11] Z. Xiao, T. qing Yang, Y. Dong and X. cai Wang, Energy harvester array using piezoelectric circular diaphragm for broadband vibration. Applied Physics Letters, 2014. 104(22): p. 223904.

[12] F. Montero de Espinosa, J. San Emeterio and P. Sanz, Summary of the measurement methods of Qm for piezoelectric materials. Ferroelectrics, 1992. 128(1): p. 61-66.

[13] G. Liu, S. Zhang, W. Jiang and W. Cao, Losses in ferroelectric materials. Materials Science and Engineering: R: Reports, 2015. 89: p. 1-48.

[14] D. L. DeVoe, Piezoelectric thin film micromechanical beam resonators. Sensors and Actuators A: Physical, 2001. 88(3): p. 263-272.

[15] G. Piazza, R. Abdolvand, G. K. Ho and F. Ayazi, Voltage-tunable piezoelectrically-transduced singlecrystal silicon micromechanical resonators. Sensors 
and Actuators A: Physical, 2004. 111(1): p. 71-78.

[16] F. Qayyum, M. Shah, S. Manzoor and M. Abbas, Comparison of thermomechanical stresses produced in work rolls during hot and cold rolling of Cartridge Brass 1101. Materials Science and Technology, 2015. 31(3): p. 317-324.

[17] F. Qayyum, M. Shah, O. Shakeel, F. Mukhtar, M. Salem and F. Rezai-Aria, Numerical simulation of thermal fatigue behavior in a cracked disc of AISI H11 tool steel. Engineering Failure Analysis, 2016. 62: p. 242-253.

[18] Z. Anjum, F. Qayyum, S. Khushnood, S. Ahmed and M. Shah, Prediction of non-propagating fretting fatigue cracks in Ti6Al4V sheet tested under pin-indovetail configuration: Experimentation and numerical simulation. Materials \& Design, 2015. 87: p. 750-758.

[19] W. T. Chien, C. J. Yang and Y. T. Yen, Coupledfield analysis of piezoelectric beam actuator using FEM. Sensors and Actuators A: Physical, 2005. 118(1): p. 171-176.

[20] K. Uchino, Y. Zhuang and S. O. URAL, Loss determination methodology for a piezoelectric ceramic: new phenomenological theory and experimental proposals. Journal of Advanced Dielectrics, 2011. 1(01): p. 17-31.

[21] R. A. Pasha and M. Z. Khan, Fatigue behavior of piezoelectric ceramic materials. Proceedings of second international conference on frontiers of advance engineering materials, 2006: p. 13-19.

[22] H. Shekhani and K. Uchino, Evaluation of the mechanical quality factor under high power conditions in piezoelectric ceramics from electrical power. Journal of the European Ceramic Society, 2015. 35(2): p. 541-544.

[23] Z. Butt and R. A. Pasha, Effect of temperature and loading on output voltage of lead zirconate titanate (PZT-5A) piezoelectric energy harvester. in IOP Conference Series: Materials Science and Engineering. 2016. IOP Publishing.

[24] Z. Butt, R. A. Pasha, F. Qayyum, Z. Anjum, N. Ahmad and H. Elahi, Generation of electrical energy using lead zirconate titanate (PZT-5A) piezoelectric material: Analytical, numerical and experimental verifications. Journal of Mechanical Science and Technology, 2016. 30(8): p. 3553-3558.

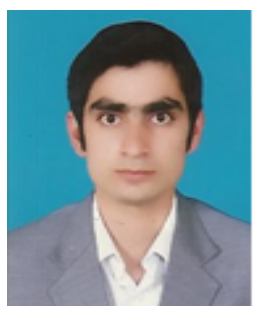

Zubair Butt He received his B.Sc. degree in Mechatronics Engineering and M.Sc. degree in Mechanical Engineering from UET Taxila, Pakistan in 2012 and 2015 respectively. He is currently working as a Lecturer in Mechatronics Engineering Department UET Taxila, Pakistan. His research activities are related to finite element analysis, simulation and characterization of piezoelectric material.

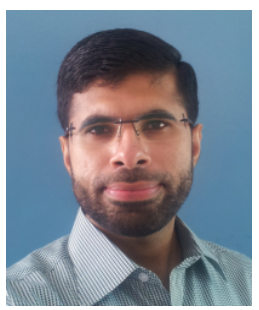

Zeeshan Anjum He received his MSc degree in mechanical engineering from University of Engineering and Technology Taxila, Pakistan in 2014. He is currently doing $\mathrm{PhD}$ from the same university. His area of specialization is fretting fatigue and tribocorrosion in titanium alloy.

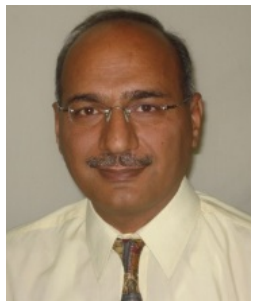

Amir Sultan He received his B.Sc. degree from UET Lahore-Pakistan in 1990, M.Sc. degree from University of Sheffield-England in 1994 and Ph.D. from UET Taxila-Pakistan in 2015. $\mathrm{He}$ is currently working as Assistant Professor in Department of Mechatronics Engineering at UET Taxila. His research interests include mechanical properties of engineering materials, alternate energy resources and multi-criteria decision making.

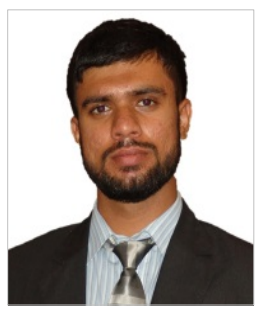

Faisal Qayyum He received both of his BSc and MSc degrees in mechanical engineering from University of Engineering and Technology Taxila, Pakistan in 2012 and 2014 respectively. $\mathrm{He}$ is currently doing $\mathrm{PhD}$ and serving as a Lecturer in the same department. His area of specialization is Applied

Mechanics and Design

Hafiz Muhammad Khurram Ali He received his degrees of B.Sc. and M.Sc. in Mechanical Engineering from UET Taxila Pakistan in 2004 and 2008 respectively. He completed his $\mathrm{PhD}$ in Industrial and Manufacturing Engineering in 2015 from the same university. He is working as Assistant Professor in Mechatronics Engineering Department of UET Taxila Pakistan. His current research interests include optimization and simulation of industrial systems and manufacturing processes.

Shahid Mehmood He received his B.Sc. Mechanical Engineering in 2004 from UET Taxila, Pakistan. He completed MSc and $\mathrm{PhD}$ in 2009 and 2016 respectively in Mechanical Engineering from same university. $\mathrm{He}$ is currently working as Assistant Professor in Mechatronics Engineering Department, UET Taxila. His research interests are material characterization and fatigue analysis. 